\title{
Astronomical Constraints on Some Long-Range Models of Modified Gravity
}

\author{
Lorenzo Iorio \\ Viale Unità di Italia 68, 70125 Bari, Italy \\ Correspondence should be addressed to Lorenzo Iorio, lorenzo.iorio@libero.it
}

Received 18 September 2007; Accepted 5 October 2007

Recommended by Sandip Pakvasa

\begin{abstract}
We use the corrections to the Newton-Einstein secular precessions of the longitudes of the perihelia of the inner planets, phenomenologically estimated E.V. Pitjeva by fitting almost one century of data with the EPM2004 ephemerides, to constrain some long-range models of modified gravity recently put forth to address the dark energy and dark matter problems. They are the fourdimensional ones obtained with the addition of inverse powers and logarithm of some curvature invariants, and the DGP multidimensional braneworld model. After working out the analytical expressions of the secular perihelion precessions induced by the corrections to the Newtonian potential of such models, we compare them to the estimated extra-rates of perihelia by taking their ratio for different pairs of planets instead of using one perihelion at a time for each planet separately, as done so far in literature. The curvature invariants-based models are ruled out, even by rescaling by a factor 10 the errors in the estimated planetary orbital parameters. Less neat is the situation for the DGP model. Only the general relativistic Lense-Thirring effect, not included, as the other exotic models considered here, by Pitjeva in the EPM force models, passes such a test.
\end{abstract}

Copyright (C) 2007 Lorenzo Iorio. This is an open access article distributed under the Creative Commons Attribution License, which permits unrestricted use, distribution, and reproduction in any medium, provided the original work is properly cited.

\section{Introduction}

Weak-field limits of theories involving long-range modifications of gravity recently put forth to address the issues of dark energy and dark matter [1-8] are important because such exotic corrections to the Newtonian potential allow, in principle, for tests to be performed on local, astronomical scales, independently of the galactic/cosmological effects [9] which motivated such alternative theories and that, otherwise, would represent their only justification. In this paper, we will show how to obtain phenomenologically tight constraints on the viability of some of such modified theories by suitably using the latest observational results from solar system planetary motions $[10,11]$. 
In Section 2, we will consider the four-dimensional models obtained from inverse powers of some curvature invariants [3]. After working out the analytic expression of the secular, that is, averaged over one orbital revolution, perihelion precession induced by the Newtonian limit of such models, we will compare it to the phenomenologically estimated corrections to the usual Newton-Einstein precessions of the perihelia of the inner planets of the solar system. By taking the ratio of them for different pairs of planets we will find that the predicted exotic effects are ruled out. In Sections 3 and 4, we repeat the same procedure for the four-dimensional model based on the logarithm of some invariants of curvature [5] and for the Dvali-Gabadadze-Porrati (DGP) multidimensional braneworld model [1], respectively, by finding that also such models do not pass our test. In Section 5, we apply the same strategy to the general relativistic gravitomagnetic field [12-14] finding that it is, instead, compatible with the ratio of the perihelion precessions for all the considered pairs of planets. Section 6 is devoted to the conclusions.

\section{The inverse-power curvature invariants models}

In this Section, we will address the long-range modifications of gravity obtained by including in the action inverse powers of some invariants of curvature not vanishing in the Schwarzschild solution [3-5]. From the correction to the Newtonian potential [3]

$$
V=-\frac{\alpha \mathrm{GM}}{r_{c}^{6 k+4}} r^{6 k+3}, \quad r \ll r_{c},
$$

where $k$ is a positive integer number, it follows a purely radial acceleration:

$$
\begin{aligned}
\mathbf{A}=-\frac{\alpha \mathrm{GM}(6 k+3)}{r_{c}^{6 k+4}} r^{6 k+2} \widehat{\mathbf{r}}, \\
r \ll r_{c} .
\end{aligned}
$$

The length scale $r_{c}$ depends, among other things, on a parameter $\mu$ which must assume a certain value in order that the model in [3] is able to reproduce the cosmic acceleration $[15,16]$ without dark energy; it is just such a value of $\mu$ which makes $r_{c} \approx 10 \mathrm{pc}(k=1)$ for a sun-like star [3]. Since [4]

$$
|\alpha|=\frac{k(1+k)}{(6 k+3) 2^{4 k} 3^{k}}
$$

and $r_{c} \approx 10 \mathrm{pc}(k=1)$, the condition $r \ll r_{c}$ for which the expansion in $r / r_{c}$ yielding (2.1) retains its validity is fully satisfied in the solar system, and (2.2) can be treated as a small correction to the Newtonian monopole with the standard perturbative techniques of celestial mechanics. The Gauss equation for the variation of the longitude of pericentre $\varpi$ of a test particle acted upon by an entirely radial disturbing acceleration $A$ is

$$
\frac{d \varpi}{d t}=-\frac{\sqrt{1-e^{2}}}{n a e} A \cos f
$$

where $a$ and $e$ are the semimajor axis and the eccentricity, respectively, of the orbit of the test particle, $n=\sqrt{\mathrm{GM} / a^{3}}$ is the unperturbed Keplerian mean motion, and $f$ is the true anomaly 
reckoned from the pericentre. The secular precession of the pericentre $\langle\dot{\varpi}\rangle$ can be worked out by evaluating the right-hand side of (2.5) onto the unperturbed Keplerian ellipse

$$
r=a(1-e \cos E)
$$

where $E$ is the eccentric anomaly, and by performing subsequently an integration over one full orbital period. To this aim, the following relations are useful:

$$
\begin{gathered}
d t=\left(\frac{1-e \cos E}{n}\right) d E, \\
\cos f=\frac{\cos E-e}{1-e \cos E} .
\end{gathered}
$$

Let us start with the case $k=1$; the extra-acceleration becomes

$$
\mathbf{A}_{k=1}=-\frac{9 \alpha \mathrm{GM}}{r_{c}^{10}} r^{8} \widehat{\mathbf{r}}
$$

By proceeding as previously outlined and using the exact result

$$
\int_{0}^{2 \pi}(\cos E-e)(1-e \cos E)^{8} d E=-\frac{5 e \pi}{64}\left[128+7 e^{2}\left(128+160 e^{2}+40 e^{4}+e^{6}\right)\right],
$$

it is possible to obtain the exact formula

$$
\langle\dot{\varpi}\rangle_{k=1}=-\frac{45 \alpha}{r_{c}^{10}} \sqrt{\mathrm{GM}^{17}\left(1-e^{2}\right)}\left[1+7 e^{2}\left(1+\frac{5}{4} e^{2}+\frac{5}{16} e^{4}+\frac{1}{128} e^{6}\right)\right] .
$$

It is important to note the dependence of $\langle\dot{\varpi}\rangle$ on a positive power of the semimajor axis $a$ : this fact will be crucial in setting our test.

The predicted extra-precession of (2.10) can be fruitfully compared to the corrections to the usual Newton-Einstein rates of the longitudes of perihelia of the inner planets of the solar system phenomenologically estimated in [10], in a least-square sense, as solve-for parameters of a global solution in which a huge amount of modern planetary data of all types covering about one century were contrasted to the dynamical force models of the EPM2004 ephemerides [11]. Such corrections are quoted in Table 1. They were determined in a modelindependent way, without modeling this or that particular model of modified gravity: only known Newton-Einstein accelerations ${ }^{1}$ were, in fact, modeled so that the estimated perihelion extra-rates account, in principle, for all the unmodeled forces present in nature. Since July 2005 [17], many other authors so far used the extra-precessions of the perihelia of the inner planets of the solar system estimated in [10] to put constraints on modified models of gravity [18-23], cosmological constant [24, 25], various cosmological issues [26-30], dark matter distribution [31-35], trans-Neptunian bodies [36], general relativity [17, 37]; a common feature of all such analyses is that they always used the perihelia separately for each planet, or combined linearly by assuming that the exotic effects investigated were included in the estimated

\footnotetext{
${ }^{1}$ With the exception of the general relativistic gravitomagnetic interaction, yielding the Lense-Thirring effect, and of the Kuiper belt objects.
} 
Table 1: Semimajor axes $a$, in AU (1 AU $\left.=1.49597870691 \times 10^{11} \mathrm{~m}\right)$, and phenomenologically estimated corrections to the Newtonian-Einsteinian perihelion rates, in arcseconds per century $\left(1 / \mathrm{cy}^{-1}\right)$, of Mercury, the Earth, and Mars [10]. Also the associated errors are quoted: they are in $m$ for $a$ [11] and in $" \mathrm{cy}^{-1}$ for $\dot{\varpi}[10]$. For the semimajor axes they are the formal, statistical ones, while for the perihelia they are realistic in the sense that they were obtained from comparison of many different solutions with different sets of parameters and observations (Pitjeva, private communication 2005). The results presented in the text do not change if $\delta a$ are rescaled by a factor 10 in order to get more realistic uncertainties.

\begin{tabular}{lcccc}
\hline Planet & $a(\mathrm{AU})$ & $\delta a(m)$ & $\dot{\varpi}\left(\prime \prime \mathrm{cy}^{-1}\right)$ & $\delta \dot{\varpi}\left(\prime \prime \mathrm{cy}^{-1}\right)$ \\
\hline Mercury & 0.38709893 & 0.105 & -0.0036 & 0.0050 \\
Earth & 1.00000011 & 0.146 & -0.0002 & 0.0004 \\
Mars & 1.52366231 & 0.657 & 0.0001 & 0.0005 \\
\hline
\end{tabular}

corrections to the perihelia precessions, and by using their errors to constrain the parameters of the extra-forces. About the reliability of the results in $[10,23]$ made an independent check by assessing the total mass of the Kuiper belt objects and getting results compatible with other ones obtained with different methods, not based on the dynamics of the inner planets. It must be noted that more robustness could be reached if and when other teams of astronomers will estimate their own corrections to the perihelion precessions. On the other hand, an alternative approach would consist in refitting the entire data set by including an ad-hoc parameter accounting for just the exotic effect one is interested in. However, such a procedure might be not only quite time-consuming because of the need of modifying the software's routines by including the extra-accelerations, but it would be also model-dependent by, perhaps, introducing the temptation of more or less consciously tweaking somehow the data and/or the procedure in order to obtain just the outcome one a priori expects.

Here we will not use one perihelion at a time for each planet. Indeed, let us consider a pair of planets $A$ and $B$ and take the ratio of their estimated extra rates of perihelia: if $(2.10)$ is responsible for them, then the quantity ${ }^{2}$

$$
\Gamma_{A B}=\left|\frac{\dot{\varpi}^{A}}{\dot{\varpi}^{B}}-\left(\frac{a^{A}}{a^{B}}\right)^{17 / 2}\right|
$$

must be compatible with zero, within the errors. The figures of Table 1 tell us that it is definitely not so: indeed, for $A=$ Mars, $B=$ Mercury, we have

$$
\Gamma_{\mathrm{MaMe}}=10^{5} \pm 0.1
$$

The situation is slightly better for $A=$ Mars and $B=$ Earth:

$$
\Gamma_{\mathrm{MaE}}=38 \pm 3.5
$$

An intermediate case occurs for $A=$ Earth and $B=$ Mercury:

$$
\Gamma_{\mathrm{EMe}}=10^{3} \pm 0.2
$$

It is important to note that the following.

\footnotetext{
${ }^{2}$ It turns out that the multiplicative term depending on the eccentricities has a negligible effect on our conclusions.
} 
(i) The uncertainty in $\Gamma_{A B}$ has been conservatively estimated as

$$
\delta \Gamma_{A B} \leq\left|\frac{\dot{\varpi}^{A}}{\dot{\varpi}^{B}}\right|\left(\frac{\delta \dot{\varpi}^{A}}{\left|\dot{\varpi}^{A}\right|}+\frac{\delta \dot{\varpi}^{B}}{\left|\dot{\varpi}^{B}\right|}\right)+\frac{17}{2}\left(\frac{a^{A}}{a^{B}}\right)^{17 / 2}\left(\frac{\delta a^{A}}{a^{A}}+\frac{\delta a^{B}}{a^{B}}\right)
$$

by linearly adding the individual terms coming from the propagation of the errors in $\dot{\varpi}$ and $a$ in (2.11); this is justified by the existing correlations among the estimated Keplerian orbital elements. ${ }^{3}$

(ii) The results presented here do not change if we rescale by a factor 10 the formal errors in the semimajor axes [11] quoted in Table 1. The same holds also for the errors in the perihelia rates which, however, are not the mere statistical ones but are to be considered as realistic, as explained in the caption of Table 1.

(iii) The constraints obtained here with (2.11) are independent of $\alpha$ and $r_{c}$; should one use (2.10) for each planet separately to constrain $r_{c}$, it turns out that, for $|\alpha|=4 \times 10^{-3}(k=1)$, $r_{c} \lesssim 4.5$ AU. Note that with such a value the condition $r \ll r_{c}$, with which (2.1) and, thus, (2.10) were derived, holds for all the inner planets.

(iv) For $k>1$, the situation is even worse because of the resulting higher powers with which the semimajor axis enters the formulas for the perihelion rates.

\section{The logarithmic curvature invariants models}

The same approach can be fruitfully used for the model in [5] based on an action depending on the logarithm of some invariants of the curvature in order to obtain a modification of gravity at the MOND [38] characteristic scale [39], so to address in a unified way the dark energy and dark matter problems; in this model the length scale $r_{c}$ amounts to about 0.04 pc for the Sun. The correction to the Newtonian potential is

$$
V \propto \frac{\mathrm{GM} r^{3}}{r_{c}^{4}}
$$

which yields the perturbing acceleration

$$
\mathbf{A} \propto \frac{r^{2}}{r_{c}^{4}} \widehat{\mathbf{r}}
$$

By using

$$
\int_{0}^{2 \pi}(\cos E-e)(1-e \cos E)^{2} d E=-e \pi\left(4+e^{2}\right),
$$

the secular precession of perihelion induced by (3.2) is

$$
\langle\dot{\varpi}\rangle \propto \frac{\sqrt{\mathrm{GM}^{5}\left(1-e^{2}\right)}}{r_{c}^{4}}\left(4+e^{2}\right) ;
$$

\footnotetext{
3 The correlations among the perihelion rates are low, with a maximum of 20\%between the Earth and Mercury (Pitjeva, private communication, 2005).
} 
also in this case, it depends on a positive power of the semimajor axis; cf. the approximated result in [5] for the shift per orbit, that is, $2 \pi\langle\dot{\varpi}\rangle / n$.

By taking the ratio of (3.4) for a pair of planets and comparing it to the ratio of the estimated extraprecessions in [10] it can be obtained

$$
\Delta_{A B}=\left|\frac{\dot{\varpi}^{A}}{\dot{\varpi}^{B}}-\left(\frac{a^{A}}{a^{B}}\right)^{5 / 2}\right| .
$$

The test is not passed. Indeed, for $A=$ Mars and $B=$ Mercury we have

$$
\Delta_{\mathrm{MaMe}}=30.7 \pm 0.1 ;
$$

the pair $A=$ Earth, $B=$ Mercury yields

$$
\Delta_{\mathrm{EMe}}=10.6 \pm 0.2
$$

while $A=$ Mars, $B=$ Earth $\Delta$ is marginally compatible with zero:

$$
\Delta_{\mathrm{MaE}}=3.4 \pm 3.5 \text {. }
$$

Note that even if the real errors in the estimated extraprecessions of perihelia were up to 10 times larger than those quoted in [10] the pair Mars-Mercury would still be able to rule out the logarithmic model in [5].

\section{The multidimensional braneworld Dvali-Gabadadze-Porrati model}

Another modified model of gravity aimed to explain the cosmic acceleration without dark matter is the multidimensional braneworld model DGP [1] which predicts, among other things, an extra rate of perihelion independent of the planetary semimajor axis ${ }^{4}[40,41]$. It is incompatible with the test of the ratio of perihelia as well, although less dramatically than the previously examined models. Indeed, by defining

$$
\Psi_{A B}=\left|\frac{\dot{\varpi}^{A}}{\dot{\varpi}^{B}}-1\right|,
$$

for $A=$ Mars, $B=$ Mercury we have

$$
\Psi_{\mathrm{MaMe}}=1.0 \pm 0.2
$$

while $A=$ Earth, $B=$ Mercury yield

$$
\Psi_{E M e}=0.9 \pm 0.2 .
$$

Errors in the determined extra rates of perihelion 5 times larger than those quoted in Table 1 would allow the DGP model to pass the test. The pair $A=$ Mars, $B=$ Earth give a result compatible with zero:

$$
\Psi_{\mathrm{MaE}}=1.5 \pm 3.5 ;
$$

the same hold for the other three combinations in which $A$ and $B$ denotes the planets with the smaller and larger semimajor axes, respectively. Until now, the DGP model was not found in disagreement with the solar system data because the perihelia were used separately for each planet [19].

\footnotetext{
4 The only dependence on the features of the planetary orbits occurs through a correction quadratic in the eccentricity $e$ (2.12) which turns out to be negligible in this case.
} 


\section{General relativistic effects: gravitomagnetism}

It maybe interesting to note that contrary to the exotic effects induced by the modified models of gravity previously examined, the Lense-Thirring effect [12-14] induced by the general relativistic gravitomagnetic field of the Sun, not modeled in [10], does pass our test based on the ratio of the perihelia. Indeed, since the Lense-Thirring perihelion precessions are proportional to a negative power of the semimajor axis, that is,

$$
\langle\dot{\varpi}\rangle \propto a^{-3}
$$

the quantity

$$
\Lambda_{A B}=\left|\frac{\dot{\varpi}^{A}}{\dot{\varpi}^{B}}-\left(\frac{a_{B}}{a_{A}}\right)^{3}\right|
$$

must be considered. It turns out that it is compatible with zero for all the six combinations which can be constructed with the data of Table 1. This result enforces the analysis in [17] in which the extra rates of the perihelia were used one at a time for each planet and linearly combined by finding the general relativistic predictions for the Lense-Thirring precessions compatible with them.

\section{Conclusions}

In this paper, we used the corrections to the Newton-Einstein secular precessions of the longitudes of perihelia of the inner planets of the solar system, estimated in [10] in a least-square sense as phenomenological solve for parameters of a global solution in which almost one century of data was fitted with the EPM2004 ephemerides, to put tight constraints on several models of modified gravity recently proposed to explain dark energy/dark matter issues. By using the ratio of the perihelion precessions for different pairs of planets, instead of taking one perihelion at a time for each planet as done so far, we were able to rule out all the considered long-range models of modified gravity, in particular the ones based on inverse powers of curvature invariants in [3] and on the logarithm of some curvature invariants [5] even by rescaling by a factor 10 the errors in the estimated perihelion extra rates. The situation is less dramatic for the DGP [1] braneworld model since if the real errors in the perihelion precessions were, in fact, 5 times larger than the ones released, it would become compatible with the data. Only the general relativistic Lense-Thirring effect passed the test. However, it must be noted that our results are based only on the extra rates of perihelia determined in [10]: it would be highly desirable to use corrections to the secular motion of perihelia independently estimated by other teams of astronomers as well. If and when they will be available, our test will become more robust.

\section{References}

[1] G. Dvali, G. Gabadadze, and M. Porrati, "4D gravity on a brane in 5D Minkowski space," Physics Letters B, vol. 485, no. 1-3, pp. 208-214, 2000.

[2] G. Allemandi, M. Francaviglia, M. L. Ruggiero, and A. Tartaglia, "Post-Newtonian parameters from alternative theories of gravity," General Relativity and Gravitation, vol. 37, no. 11, pp. 1891-1904, 2005. 
[3] I. Navarro and K. van Acoleyen, "On the Newtonian limit of generalized modified gravity models," Physics Letters B, vol. 622, no. 1-2, pp. 1-5, 2005.

[4] I. Navarro and K. van Acoleyen, "Consistent long distance modification of gravity from inverse powers of the curvature," Journal of Cosmology and Astroparticle Physics, vol. 2006, no. 3, 2006, 008.

[5] I. Navarro and K. van Acoleyen, "Modified gravity, dark energy and modified Newtonian dynamics," Journal of Cosmology and Astroparticle Physics, vol. 2006, no. 9, 2006, 006.

[6] P. S. Apostolopoulos and N. Tetradis, "Late acceleration and $w=-1$ crossing in induced gravity," Physical Review D, vol. 74, no. 6, Article ID 064021, 8 pages, 2006.

[7] S. Capozziello, V. F. Cardone, and M. Francaviglia, " $f(R)$ theories of gravity in the Palatini approach matched with observations," General Relativity and Gravitation, vol. 38, no. 5, pp. 711-734, 2006.

[8] S. Nojiri and S. D. Odintsov, "Introduction to modified gravity and gravitational alternative for dark energy," International Journal of Geometric Methods in Modern Physics, vol. 4, no. 1, pp. 115-145, 2007.

[9] S. Capozziello, "Dark energy models toward observational tests and data," International Journal of Geometric Methods in Modern Physics, vol. 4, no. 1, pp. 53-78, 2007.

[10] E. V. Pitjeva, "Relativistic effects and solar oblateness from radar observations of planets and spacecraft," Astronomy Letters, vol. 31, no. 5, pp. 340-349, 2005.

[11] E. V. Pitjeva, "High-precision ephemerides of planets-EPM and determination of some astronomical constants," Solar System Research, vol. 39, no. 3, pp. 176-186, 2005.

[12] J. Lense and H. Thirring, "Über den einfluss der eigenrotation der zentral körper auf die bewegung der planeten und monde nach der einsteinschen gravitationstheorie," Physikalische Zeitschrift, vol. 19, pp. 156-163, 1918.

[13] B. Mashhoon, F. W. Hehl, and D. S. Theiss, "On the gravitational effects of rotating masses: the Thirring-Lense papers," General Relativity and Gravitation, vol. 16, no. 8, pp. 711-750, 1984.

[14] R. J. Ruffini and C. Sigismondi, Eds., Nonlinear Gravitodynamics, World Scientific, Singapore, 2003.

[15] S. M. Carroll, A. de Felice, V. Duvvuri, D. A. Easson, M. Trodden, and M. S. Turner, "Cosmology of generalized modified gravity models," Physical Review D, vol. 71, no. 6, Article ID 063513, 11 pages, 2005.

[16] O. Mena, J. Santiago, and J. Weller, "Constraining inverse-curvature gravity with supernovae," Physical Review Letters, vol. 96, no. 4, Article ID 041103, 4 pages, 2006.

[17] L. Iorio, "First preliminary tests of the general relativistic gravitomagnetic field of the Sun and new constraints on a Yukawa-like fifth force from planetary data," Planetary and Space Science, vol. 55, no. 10, pp. 1290-1298, 2007.

[18] R. Gannouji, D. Polarski, A. Ranquet, and A. A. Starobinsky, "Scalar-tensor models of normal and phantom dark energy," Journal of Cosmology and Astroparticle Physics, vol. 2006, no. 9, 2006, 016.

[19] L. Iorio and G. Giudice, "On the perspectives of testing the Dvali-Gabadadze-Porrati gravity model with the outer planets of the Solar System," Journal of Cosmology and Astroparticle Physics, vol. 2006, no. $08,2006,007$.

[20] R. H. Sanders, "Solar system constraints on multifield theories of modified dynamics," Monthly Notices of the Royal Astronomical Society, vol. 370, no. 3, pp. 1519-1528, 2006.

[21] G. S. Adkins and J. McDonnell, "Orbital precession due to central-force perturbations," Physical Review D, vol. 75, no. 8, Article ID 082001, 7 pages, 2007.

[22] M. L. Ruggiero and L. Iorio, "Solar System planetary orbital motions and $f(R)$ theories of gravity," Journal of Cosmology and Astroparticle Physics, vol. 2007, no. 1, 2007, 010.

[23] L. Iorio, "Constraints on the range $\lambda$ of Yukawa-like modifications to the Newtonian inverse-square law of gravitation from solar system planetary motions," Journal of High Energy Physics, vol. 2007, no. $10,2007,041$.

[24] L. Iorio, "Can solar system observations tell us something about the cosmological constant?" International Journal of Modern Physics D, vol. 15, no. 4, pp. 473-475, 2006.

[25] P. Jetzer and M. Sereno, "Two-body problem with the cosmological constant and observational constraints," Physical Review D, vol. 73, no. 4, Article ID 044015, 6 pages, 2006.

[26] G. S. Adkins, J. McDonnell, and R. N. Fell, "Cosmological perturbations on local systems," Physical Review D, vol. 75, no. 6, Article ID 064011, 9 pages, 2007.

[27] S. Fay, S. Nesseris, and L. Perivolaropoulos, "Can $f(R)$ modified gravity theories mimic a $\Lambda$ CDM cosmology?" Physical Review D, vol. 76, no. 6, Article ID 063504, 10 pages, 2007.

[28] S. Nesseris and L. Perivolaropoulos, "Crossing the phantom divide: theoretical implications and observational status," Journal of Cosmology and Astroparticle Physics, vol. 2007, no. 1, 2007, 018. 
[29] S. Nesseris and L. Perivolaropoulos, "Limits of extended quintessence," Physical Review D, vol. 75, no. 2, Article ID 023517, 9 pages, 2007.

[30] M. Sereno and P. Jetzer, "Evolution of gravitational orbits in the expanding universe," Physical Review $D$, vol. 75, no. 6, Article ID 064031, 8 pages, 2007.

[31] L. Iorio, "Solar system planetary orbital motions and dark matter," Journal of Cosmology and Astroparticle Physics, no. 5, pp. 2, 2006.

[32] I. B. Khriplovich and E. V. Pitjeva, "Upper limits on density of dark matter in solar system," International Journal of Modern Physics D, vol. 15, no. 4, pp. 615-618, 2006.

[33] M. Sereno and P. Jetzer, "Dark matter versus modifications of the gravitational inverse-square law: results from planetary motion in the solar system," Monthly Notices of the Royal Astronomical Society, vol. 371, no. 2, pp. 626-632, 2006.

[34] J.-M. Frére, F.-S. Ling, and G. Vertongen, "Bound on the dark matter density in the solar system from planetary motions," preprint, 2007, http://arxiv.org/abs/astroph/0701542v1.

[35] I. B. Khriplovich, "Density of dark matter in solar system and perihelion precession of planets," International Journal of Modern Physics D, vol. 16, no. 9, pp. 1475-1478, 2007.

[36] L. Iorio, "Dynamical determination of the mass of the Kuiper Belt from motions of the inner planets of the solar system," Monthly Notices of the Royal Astronomical Society, vol. 375, no. 4, pp. 1311-1314, 2007.

[37] C. M. Will, "The confrontation between general relativity and experiment," Living Reviews in Relativity, vol. 9, pp. 1-100, 2006.

[38] M. Milgrom, "A modification of the Newtonian dynamics as a possible alternative to the hidden mass hypothesis," Astrophysical Journal, vol. 270, pp. 365-370, 1983.

[39] R. H. Sanders and S. S. McGaugh, "Modified Newtonian dynamics as an alternative to dark matter," Annual Review of Astronomy and Astrophysics, vol. 40, pp. 263-317, 2002.

[40] A. Lue and G. Starkman, "Gravitational leakage into extra dimensions: probing dark energy using local gravity," Physical Review D, vol. 67, no. 6, Article ID 064002, 9 pages, 2003.

[41] L. Iorio, "On the effects of Dvali-Gabadadze-Porrati braneworld gravity on the orbital motion of a test particle," Classical and Quantum Gravity, vol. 22, no. 24, pp. 5271-5281, 2005. 

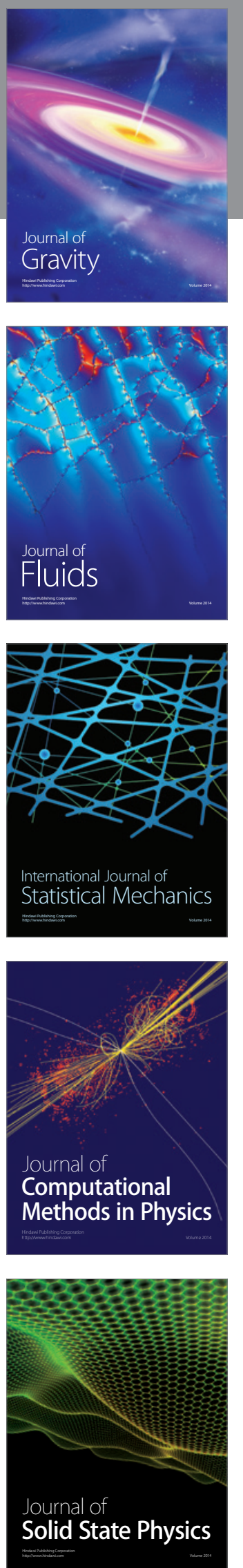

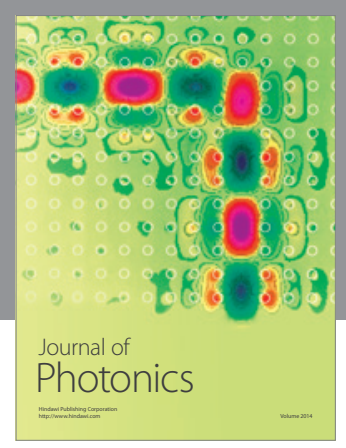

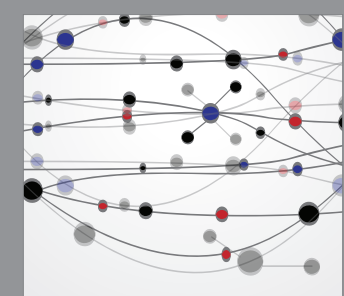

The Scientific World Journal
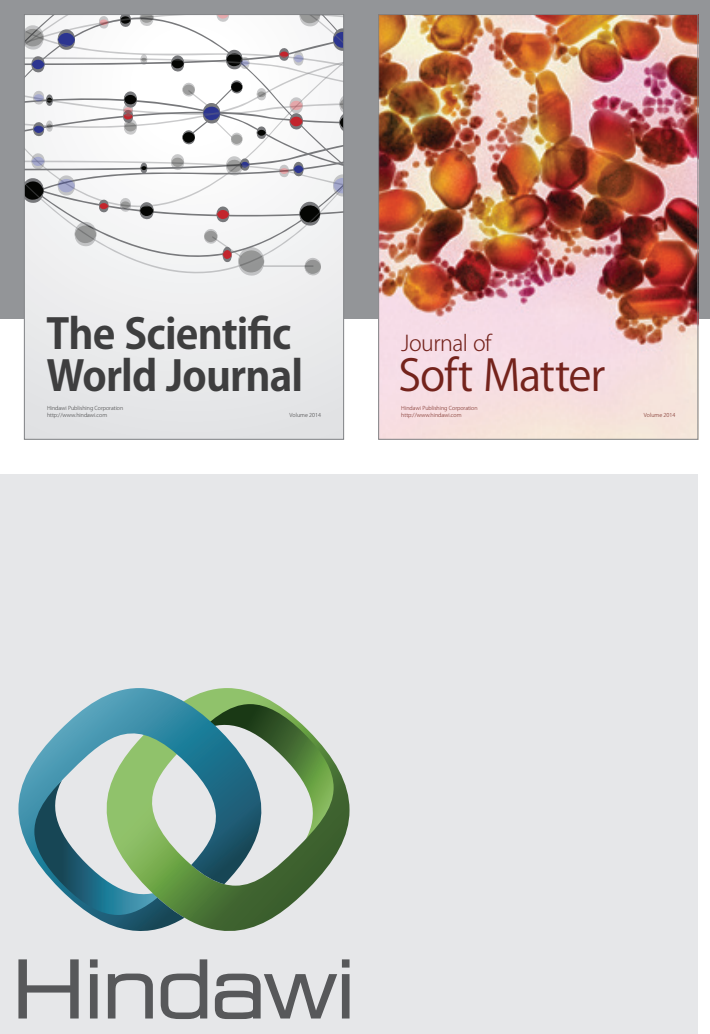

Submit your manuscripts at

http://www.hindawi.com
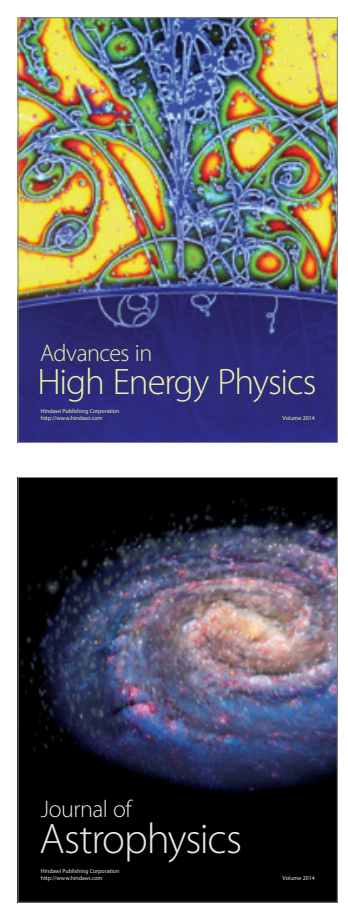
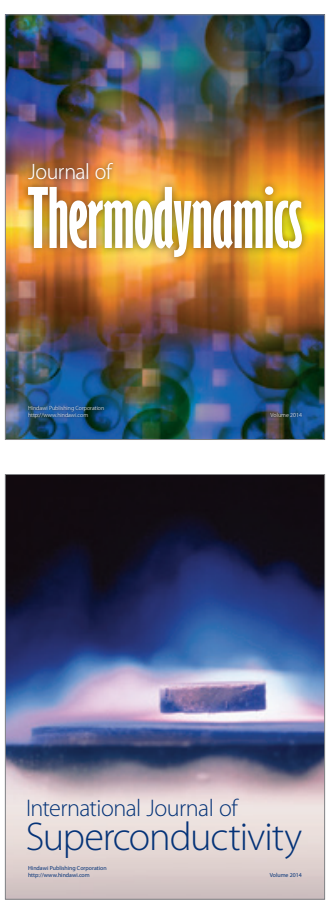
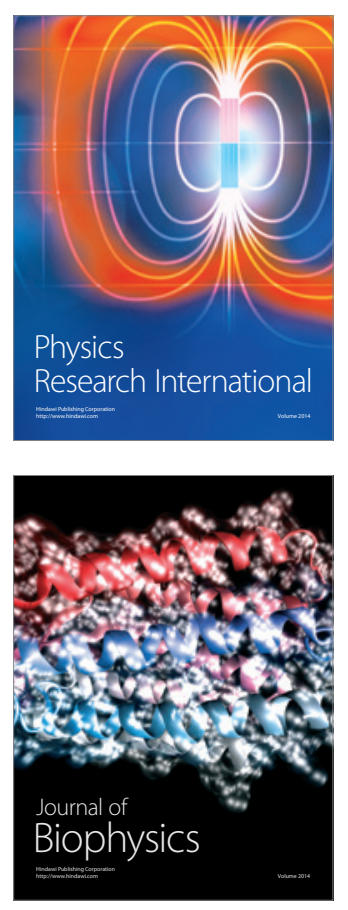
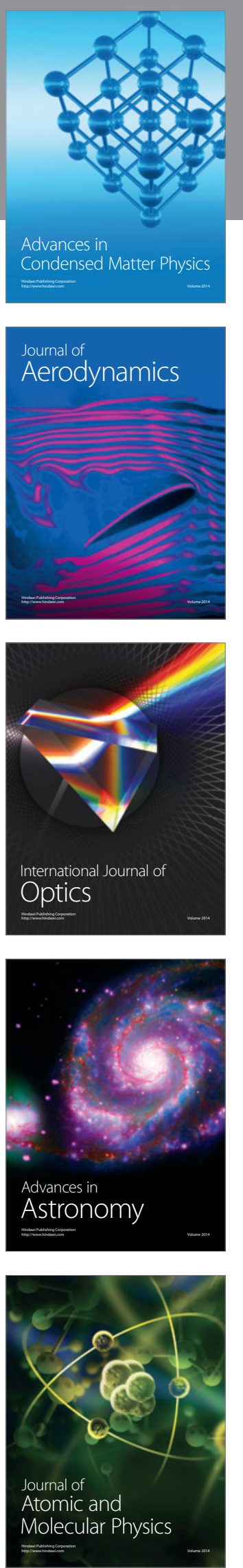